OSAHS

\title{
Evaluation of neurocognitive abilities in children affected by obstructive sleep apnea syndrome before and after adenotonsillectomy
}

\author{
Valutazione delle abilità neurocognitive in bambini affetti da sindrome delle apnee \\ ostruttive in sonno prima e dopo adenotonsillectomia
}

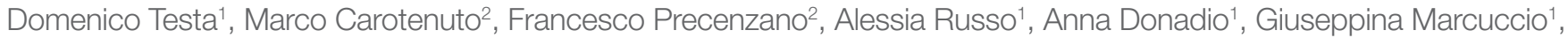 \\ Gaetano Motta' ${ }^{1}$
}

'Otolaryngology, Head and Neck Surgery, Department of General and Specialistic Surgery, University of Campania "Luigi Vanvitelli", Italy; ${ }^{2}$ Clinic of Child and Adolescent Neuropsychiatry, Department of Mental Health, Physical and Preventive Medicine, University of Campania "Luigi Vanvitelli", Italy

\section{SUMMARY}

Obstructive sleep apnoea syndrome (OSAS) is the most severe form of sleep-related disordered breathing (SRDB) and is characterised by snoring, apnoeas, and/or hypopnoeas associated to hypoxia, hypercarbia, or repeated arousals from sleep. OSAS has three major categories of morbidities: neurobehavioural, cardiovascular and somatic growth failure. The gold standard for objective diagnosis of obstructive-SRDB severity is polysomnography (PSG). The indication for surgical treatment in children is moderate-severe OSAS (AHI, apnoea hypopnoea index $>5 / \mathrm{h}$ ) and in mild OSAS (AHI 2-5/h) with complications or morbidity. The entire spectrum of PSG-defined SRDB (ranging from Primary Snoring to severe OSAS) may correlate with behavioural, attentional and executive function deficits relating to hypoxia and sleep disruption: in some cases, these alterations may mimic attention deficit hyperactivity disorder (ADHD). The aim of this research was to evaluate visuoperceptual and constructional abilities, paediatric sleep questionnaire and polysomnographic scores before and 6 months after adenotonsillectomy with objective and subjective information. We included 59 children who underwent neuropsychiatric and otolaryngologist clinical evaluation and the Beery-Buktenica Developmental Test of Visual-Motor Integration (VMI); children parents were asked to fill in the Paediatric Sleep Questionnaire (PSQ); each child underwent PSG. At 6 months after adenotonsillectomy, all patients were evaluated again. There is a significant difference in PSQ parameters, VMI standard, visual tests scores and PSG parameters before and after adenotonsillectomy in children affected by OSAS. These results showed the achievement of therapeutic benefits with improvement of the quality of life for both children and their parents.

KEY WORDS: OSAS, adenotonsillectomy, pediatric OSAS, neuropsychological nonverbal skills

\section{RIASSUNTO}

La Sindrome delle Apnee Ostruttive del Sonno (OSAS, Obstructive Sleep Apnea Syndrome) è la forma più severa di Disturbo Respiratorio Sonno-correlato (SRDB, Sleep-related Disordered Breathing) ed è caratterizzata da russamento, apnee, elo ipopnee associate a ipossia, ipercapnia, o ripetuti risvegli dal sonno (arousals). L'OSAS include tre categorie di morbidità: neurocomportamentali, cardiovascolari, disturbi della crescita somatica. Il gold standard per la diagnosi oggettiva di severità del SRDB ostruttivo è rappresentato dalla polisonnografia (PSG). L'indicazione al trattamento chirurgico nei bambini è la diagnosi di OSAS moderata-severa (indice di apnea-ipopnea $>5 / h$ ) e di OSAS lieve (indice di apneaipopnea $2-5 / h$ ) se sono presenti complicanze o comorbidità. Lo spettro intero di SRDB definiti mediante PSG (dal Russamento Primario all'OSAS severa) potrebbe correlare con deficit comportamentali, attentive e delle funzioni esecutive dovuti all'ipossia ed alla frammentazione del sonno: in alcuni casi, queste alterazioni possono mimare il Disturbo da deficit di Attenzione con Iperattività (ADHD, Attention Deficit Hyperactivity Disorder). Lo
Received: May 2, 2019

Accepted: Juy 28, 2019

\section{Correspondence}

Giuseppina Marcuccio

Via Fontana 7, 81010 Castel Campagnano (CE), Italy

E-mail: giuseppina_marcuccio@hotmail.it

\section{Funding}

None.

Conflict of interest

The Authors declare no conflict of interest.

\begin{abstract}
How to cite this article: Testa D, Carotenuto M, Precenzano F, et al. Evaluation of neurocognitive abilities in children affected by obstructive sleep apnea syndrome before and after adenotonsillectomy. Acta Otorhinolaryngol Ital 2020;40:122-132.https://doi.org/10.14639/0392$100 \mathrm{X}-\mathrm{N} 0267$
\end{abstract}

(C) Società Italiana di Otorinolaringoiatria e Chirurgia Cervico-Facciale

\section{(c) (1) () $)$}

This is an open access article distributed in accordance with the CC-BY-NC-ND (Creative Commons Attribution-NonCommercial-NoDerivatives 4.0 International) license. The article can be used by giving appropriate credit and mentioning the license, but only for non-commercial purposes and only in the original version. For further information: https:// creativecommons.org/licenses/by-nc-nd/4.0/deed.en 
scopo di questa ricerca è stato quello di valutare le abilità visuopercettive e costruttive, i punteggi al Pediatric Sleep Questionnaire ed alla PSG prima e dopo l'intervento di adenotonsillectomia fornendo informazoni oggettive e soggettive. Abbiamo incluso in questo studio 59 bambini sottoposti a valutazione clinica otorinolaringoiatrica e neuropsichiatrica e poi al test di Beery-Buktenica Developmental Test of Visual-Motor Integration (VMI) ed ai genitori veniva chiesto di compilare il Pediaric Sleep Questionnaire (PSQ); ogni bambino veniva sottoposto a PSG. Dopo 6 mesi dall'intervento di adeotonsillectomia sono state ripetute tutte le valutazioni come al tempo 0. L'analisi statistica evidenzia risultati significativi al PSQ, VMI standard e visivo ed ai parametri polisonnografici se confrontati prima e dopo l'intervento chirurgico di adenotonsillectomia nei bambini affetti da OSAS moderata-severa. Questi risultati hanno mostrato il raggiungimento dei vantaggi terapeutici attraverso il miglioramento della qualità della vita dei bambini e dei loro genitori.

PAROLE CHIAVE: OSAS, adenotonsillectomia, OSAS pediatrica, abilità neuropsicologiche non verbali

\section{Introduction}

Sleep-related disordered breathing (SRDB), pathologic nocturnal respiratory functioning, includes clinical conditions that range from primary snoring (PS) to obstructive sleep apnoea syndrome (OSAS) and represents one of the most common sleep disorders in childhood affecting up to one-third of children (prevalence up to $34.5 \%)^{1,2}$.

OSAS is believed to be present in about $1 \%$ to $3 \%$ in children aged 2 to 18 with no gender predominance; furthermore, chronic snoring may be present in more than $10 \%$ of children ${ }^{3-5}$. PS and OSAS are the two extreme conditions of a wide spectrum of increased resistance in the upper airways ${ }^{3-5}$.

PS is the mildest form of SRDB and is defined as habitual snoring without discrete respiratory events, gas exchange abnormalities, or evidence of sleep fragmentation; OSAS is the most severe form of SRDB and is characterised by snoring, apneoas, and/or hypopnoeas associated with hypoxia, hypercarbia, or repeated arousals from sleep ${ }^{6}$.

OSAS has three major categories of morbidities: neurobehavioural, cardiovascular and somatic growth failure 7,8 . Symptoms in children with OSAS include snoring, breathing difficulty and/or breathing pauses during sleep, excessive sweating and enuresis; daytime symptoms may include oral breathing, headaches and excessive sleepiness, behavioural and neurocognitive changes such as attention deficit, hyperactivity, irritability, learning difficulties, memory loss and intelligence alterations ${ }^{9-12}$. In adults but less so in children, OSAS is associated with arterial systemic hypertension due to alterations in the renin-angiotensin axis secondary to hypoxia during sleep; left ventricular wall thickness; pulmonary vascular hypertension ${ }^{13,14}$. The gold standard for diagnosing OSAS is overnight polysomnography (PSG) which gives information about the frequency and severity of respiratory events and associated blood gas changes ${ }^{15}$.

The European Respiratory Society (ERS) Task Force on diagnosis and management of obstructive SRDB in childhood (2 to 18 years old) analysed selected evidence from 362 articles since prospective cohort studies describing its clinical topics and randomised, double-blind, placebocontrolled trials, regarding its treatments, are scarce ${ }^{16}$. Kaditis et al. summarised the conclusions of the ERS Task Force indicating seven steps for diagnosis and management of SRDB in children (Tab. I) ${ }^{16,17}$.

Some clinical conditions can be considered as factors predicting long-term persistence of obstructive SRDB: obesity and increasing BMI percentile, male sex, severity of OSAS (AHI > 5 episodes/h), African-American Ethnicity and persistent tonsillar hypertrophy with a narrow mandible ${ }^{18,19}$. The gold standard for objective diagnosis of obstructiveSRDB severity is PSG and is indicated in children candidate to adenotonsillectomy especially in the presence of obesity, craniofacial deformities, neuromuscular disorders, complex abnormalities such as Chiari malformation, Down syndrome and Prader-Willi syndrome or when the need for treatment is unclear ${ }^{20,21}$. Furthermore, PSG is indicated before and after rapid maxillary expansion or application of oral appliances, continuous positive airway pressure (CPAP) or noninvasive positive pressure ventilation (NPPV) treatments and when symptoms of OSAS persist after therapy ${ }^{20}$.

The AHI, or the number of mixed, obstructive or central apnoeas and hypopnoeas per hour of total sleep time, is the most commonly used parameter to describe the severity of SRDB ${ }^{22}$.

In children without SRDB symptoms or associated morbidities, the $90^{\text {th }}$ percentile for the AHI according to the American Academy of Sleep Medicine (AASM 2007 scoring rules) is 3.2 episodes/h for the second year of life, up to 2.5 episodes/h for the ages $>2$ and $\leq 6$ years, and up to 2.1 episodes/h for the ages $>6$ and $<18$ years ${ }^{23}$. OSAS is defined when AHI $\geq 2$ episodes/h or in presence of SRDB symptoms when AHI $\geq 1$ episode/h: mild OSAS if AHI 2-5 episodes/h; moderate-severe OSAS if AHI $>5$ episodes/h (Tab. II) ${ }^{18,23}$.

Indications for treatment in children is moderate-severe OSAS (AHI $>5 / \mathrm{h}$ ) or mild OSAS (AHI 2-3/h) only when morbidities are present ${ }^{18}$. The need for combined treatments (adenotonsillectomy when needed, weight loss, CPAP, 
D. Testa et al.

Table I. A stepwise approach to the diagnosis and management of obstructive SRDB in 2-18-year-old children (from Kaditis et al., $2016{ }^{16}$; Kaditis et al., $2012^{17}$ ).

\section{Step 1 Child at risk of SDB if (one or more)}

1.1. Symptoms of upper airway obstruction (snoring, apnoea, restless sleep, oral breathing)

1.2. Finding on exam (tonsillar hypertrophy, obesity, midface deficiency, mandibular hypoplasia, neuromuscular disorders, Down syndrome, Prader-Willi Syndrome)

1.3. Objective findings related to SDB (lateral neck radiography, flexible nasopharyngoscopy, cephalometry, upper airway MRI or CT)

1.4. Prematurity or family history of SDB

\section{Step 2 Recognition of morbidity and conditions coexisting with SRDB}

2.1. Morbidity

- Cardiovascular system:

- elevated blood pressure

- pulmonary hypertension and cor pulmonale

- Central nervous system:

- excessive daytime sleepiness

- inattention/hyperactivity

- cognitive deficits/academic difficulties

- behavioural problems

- enuresis and somatic growth delay or growth failure

- decreased quality of life

- Conditions coexisting with SRDB (probably common pathogenesis):

- history of recurrent otitis media or tympanostomy tube placement

- recurrent wheezing or asthma

- metabolic syndrome

- oral-motor dysfunction

\section{Step 3 Recognition factors predicting long-term persistence of SDB}

\section{1 .}

- Obesity and increasing BMI percentile

- Male sex

- Obstructive AHI > 5 episodes/h

- African-American ethnicity

- Untreated tonsillar hypertrophy, narrow mandible

Step 4 Objective diagnosis and assessment of SDB severity

4.1. PSG or polygraphy if child at risk for SDB (step 1 and 2)

4.2.

- OSAS-definition 1: SDB symptoms in combination with obstructive AHI $\geq 2$ episodes/h or obstructive apnea index $\geq 1$ episodes/h

- OSAS-definition 2: SDB symptoms and AHI $\geq 1$ episodes/h (including central events)

4.3. If $A H I \geq 5$ episodes/h SDB unlikely to resolve spontaneously and child at risk for morbidity

4.4. if PSG or polygraphy not available: ambulatory PSG or polygraphy, nocturnal oximetry, Paediatric Sleep Questionaire or Sleep Clinical Record

\section{Step 5 Indication for treatment of SDB}

5.1 .

- $\mathrm{AHI}>5$ episodes/h irrespective of the presence of morbidity

- Treatment may be beneficial if AHI 1-5 episodes/h especially in the presence of morbidity from the cardiovascular system (see 2.1.); from the central nervous system (see 2.1.); enuresis; somatic growth delay or growth failure; decreased quality of life; risk factors for SDB persistence (see step 3)

- If at risk for SDB and PSG or polygraphy not available, treatment is considered when positive oximetry or SDB questionnaire (see 4.4.) or morbidity present

5.2. Unclear whether should treat primary snoring (evaluation annually)

5.3. OSAS treatment is a priority in the presence of: major craniofacial abnormalities; neuromuscular disorders; achondroplasia; Chiari malformation; Down syndrome; mucopolysaccharidoses; Prader-Willi syndrome

\section{Step 6 Stepwise treatment approach to SDB}

6.1. A stepwise treatment approach (from 6.2. to 6.9.) is usually implemented until complete resolution of SDB

6.2. Weight loss if the child is overweight or obese

6.3. Nasal corticosteroids and/or montelukast

6.4. Adenotonsillectomy

6.5. Unclear whether adenoidectomy or tonsillectomy alone are adequate

6.6. Rapid maxillary expansion and orthodontic appliances

6.7. CPAP or NPPV (for nocturnal hypoventilation)

6.8. Craniofacial surgery

6.9. Tracheostomy 
Table I. Follows.

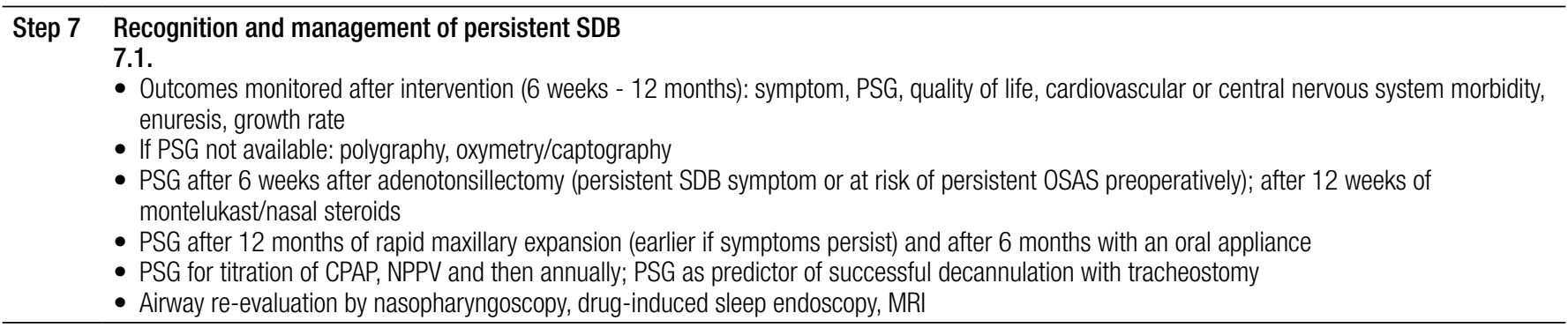

MRl: magnetic resonance imaging; CT: computed tomography; BMI: body mass index; AHI: apnoea-hypopnoea index; PSG: polysomnography; OSAS: obstructive sleep apnoea syndrome; CPAP: continuous positive airway pressure; NPPV: noninvasive positive pressure ventilation.

Table II. Definition of OSAS from AHI parameter (from Marcus et al., $2013^{18}$; lber et al., $2007^{23}$ ).

\begin{tabular}{ll}
$\begin{array}{l}\text { Normal values or OSAS } \\
\text { definition }\end{array}$ & AHI (episodes/h) \\
$\begin{array}{l}\text { No SRDB symptoms } \\
\text { or morbidity }\end{array}$ & $3.2 \mathrm{AHI}\left(2\right.$ years old) $\left(90^{\text {th }}\right.$ percentile) \\
& $2.5 \mathrm{AHI}\left(2-6\right.$ years old) $\left(90^{\text {th }}\right.$ percentile) \\
& $2.1 \mathrm{AHI}$ (6-18 years old) $\left(90^{\text {th }}\right.$ percentile) \\
SRDB symptoms & \\
and/or morbidity & \\
Mild OSAS & $2-5 \mathrm{AHI}$ \\
Moderate-severe OSAS & $>5 \mathrm{AHI}$ \\
\hline
\end{tabular}

NPPV) is a priority in these clinical conditions: major craniofacial abnormalities, neuromuscular disorders (i.e. Duchenne muscular dystrophy), achondroplasia, Chiari malformation, Down syndrome, mucopolysaccharidoses, Prader-Willi syndrome ${ }^{24-26}$.

The entire spectrum of PSG-defined SRDB (ranging from PS to severe OSAS) may correlate with behavioural, attentional and executive function deficits relating to hypoxia and sleep disruption ${ }^{27}$.

It is important to emphasise the risk of greater impairment of both behavioural and cognitive functions in children with milder forms of SRDB so that PS is not a universally benign condition ${ }^{28}$. Preschool children with obstructive SRDB are more vulnerable in their adaptative and behavioural function vs. cognitive function: this age has a sort of 'window of opportunity' for early treatment by preventing cognitive deficits arising later in childhood ${ }^{28}$.

Some authors analysed neurocognitive function in children with OSAS after CPAP-treatment (5-6 months): significant improvements in their abilities to analyse and synthesise abstract information, multitasking, simultaneous processing, divided attention and in recognition and recall for visual information were all shown ${ }^{29}$.

Sleep disruption, even without respiratory compromise, may induce neurocognitive alterations, and for this reason the severity of SRDB, from PS to OSAS, does not correlate with neurocognitive deficits ${ }^{30}$.

Cognitive functions are correlated with sleep fragmentation and thus to SRDB: arousals seem to be an important defensive mechanism against sleep fragmentations induced by SRDB ${ }^{31}$. Children with high levels of arousal seem to present a high degree of protection against cognitive consequences of SRDB ${ }^{32}$.

The aim of the present study is to analyse changes in neurocognitive and behavioural functions in children 4 to 11 years old affected by moderate-severe OSAS, after surgical treatment with adenotonsillectomy to investigate the role of obstruction in SRDB symptoms.

We evaluated visuoperceptual and constructional abilities, PSQ and full overnight polysomnographic values, before and 6 months after adenotonsillectomy, giving objective and subjective information about sleep disturbances in OSAS.

\section{Materials and methods}

We enrolled 86 children with obstructive SRBD, aged between 4 to 11 years old, referred by primary care paediatricians for snoring and suspected apnoeas to the Clinic of Child and Adolescent Neuropsichiatry and then to the Ear Nose and Throat Unit of the University of Campania "Luigi Vanvitelli" between October 2015 and April 2018.

Each child underwent neuropsychiatric and otolaryngologic clinical evaluation and VMI test, and parents were asked to fill in the PSQ.

Three overnight full sleep PSG were performed, and obstructive AHI were identified as normal, mild, moderate, or severe OSAS.

Inclusion criteria were: history of habitual snoring and/or apnea; frequent and continuous breathing pauses referred by parents; nocturnal difficult breathing; daytime hyperactivity; attentive deficit, poor school performance. Moreover, children with moderate (respiratory disturbance index of 5-10 episodes/h with average $\mathrm{SaO}_{2}>95 \%$ ) or severe (respiratory disturbance index $>10$ episodes/h with an average $<95 \% \mathrm{SaO}_{2}$ ) OSAS were included; tonsillar and adenoidal grading 3-4, clinical febrile episodes (FE) and frequent acute pharyngotonsillitis ${ }^{2}$; age between 4 and 11 years.

Children were excluded if they had any of the following: sensorineural hearing loss; tube-tympanic alterations (OME 
otitis media with effusion, acute otitis media recurrent AOM); nasal obstruction due to nasal septal deviation or hypertrophy of inferior and middle turbinates, obesity $\left(\mathrm{BMI} \geq 90^{\text {th }}\right.$ percentile); cardiac and pulmonary metabolic disorders; craniofacial anomalies; neuromuscular disorders and genetic syndromes.

During the first step of enrollment, we excluded 27 children:

- 15 did not meet inclusion criteria (4 obese children, BMI $\geq 90^{\text {th }}$ percentile; 2 affected by otitis media with effusions associated to conductive hearing loss; 1 affected by craniofacial anirmalities, Pierre Robin Sequence and 8 children affected by mild OSA $(\mathrm{AHI}<2)$;

- 10 children declined to participate;

- 2 children for other reasons.

At the end of this evaluation, we included 59 children affected by moderate-severe OSA (AHI > 5) with adenotonsillar hypertrophy.

The second step of treatment included adenotonsillectomy performed in the Ear Nose and Throat Unit of University of Campania "Luigi Vanvitelli".

The third step included follow-up at 6 months after surgery: children underwent sleep PSG, VMI test and their parents filled in the PSQ. During this step, 22 patients refused to continue.

The last step of the analysis of data included 37 children mean age $8.44 \pm 2.26$ (Fig. 1).

\section{Paediatric Sleep Questionnaire}

PSQ is a SRBD scale questionnaire for children parents containing 22 items, indicating the presence of apnoeas. These items regard frequency, loud snoring, observed apnoeas, alterations in breathing during sleep, daytime symptoms and signs such as sleepiness, inattentive and hyperactive behaviour. Responses are "yes" (= 1 score), "no" (= 0 score), and "do not know" (= missing). A cut-off value of 0.33 , which would be most effective in identifying pediatric OSA, was used.

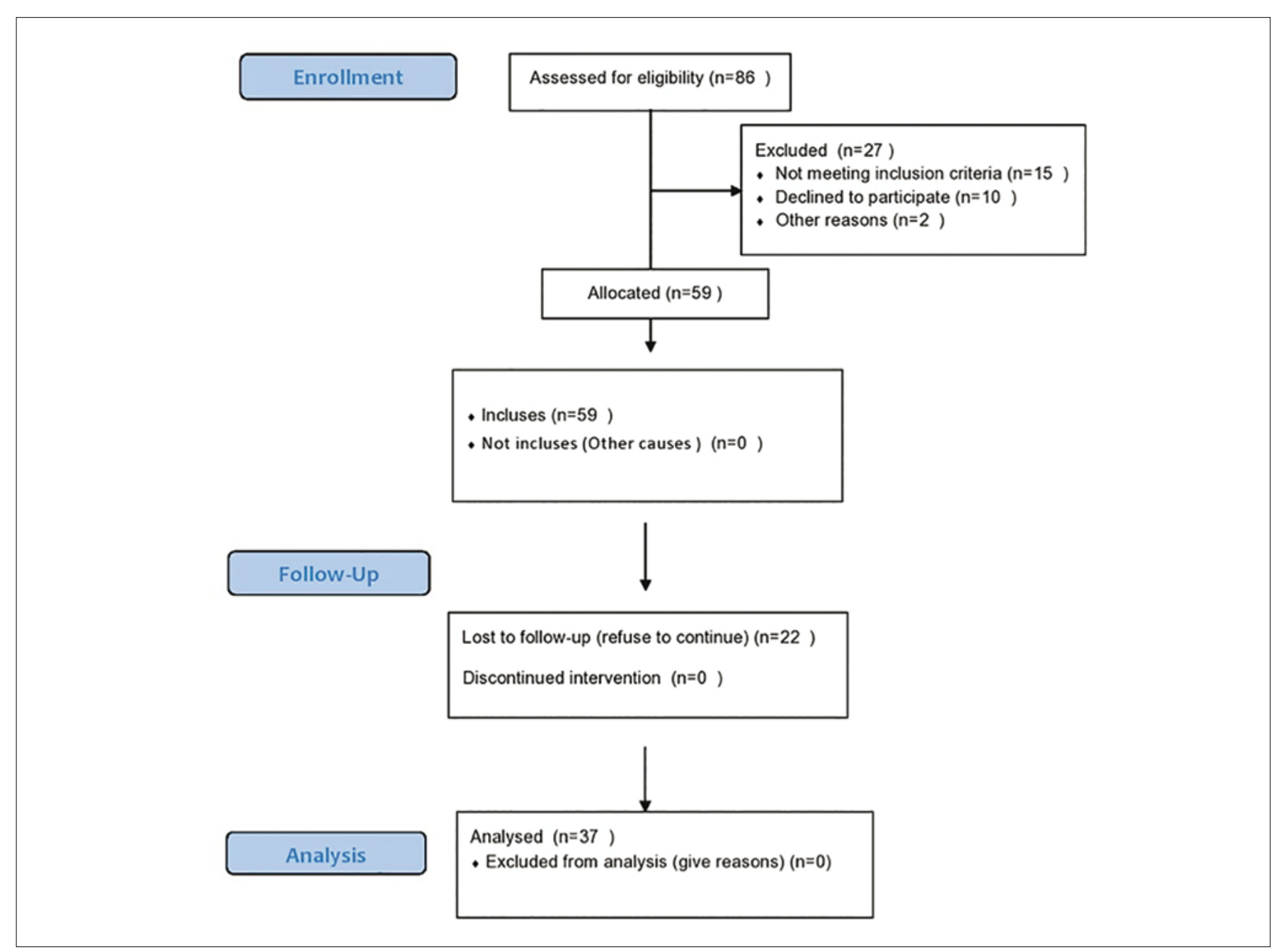

Figure 1. Enrollment and study flow. 
Beery-Buktenica Developmental Test of Visual-Motor Integration (VMI)

The Beery-Buktenica Developmental Test of Visual-Motor Integration Performances in Children (VMI task) is a paperand-pencil test in which children have to imitate or copy up to 27 geometric forms with increasing complexity using paper and pencil ${ }^{32}$. The test was stopped when a child made more than two errors in a row ${ }^{32}$. Copying errors were marked if they reflected problems in fine motor coordination and a pure visuospatial problem. The Beery VMI task is specifically designed for children and takes about 10 minutes to complete ${ }^{32}$. The Beery VMI scores were standardised for age and gender using normative data for the Italian general population ${ }^{32}$.

The percentile scores were used for diagnosing the visuomotor abnormalities in our sample. A value less than or equal to the $5^{\text {th }}$ percentile was considered to indicate VMI impairment.

\section{Overnight full polysomnography recordings (PSGs)}

The polysomnographic recording was performed using a videorecorder connected to probes, electrodes and bands suitable to the patients' age and weight. The exam is computerised and uses the Embletta System, a system recognised and appointed by the American Academy of Sleep Medicine for the study of sleep apnoea. The recording time is 6-8 hours, and supervised by medical and technical staff, who interpreted the data collected. We analysed sleep and respiratory parameters:

- Sleep Latency, SL (min);

- REM Latency, RL (min);

- Total Sleep Time, TST (min);

- Sleep Period Time, SPT or TIB-SL (min);

- Wake After Sleep Onset, WASO (min);

- 1-2-3 NREM, N1-N2-N3 (\%TST);

- REM (\%TST);

- Sleep Efficiency, SE or TST/TIB\% (\%);

- Arousal Index, AI (n Arousal/h sleep);

- Periodic Limb Movement Index, PLMI (n PLM/h Sleep);

- AHI (number of AH/h sleep)

- Nadir $\mathrm{SaO}_{2}$ during sleep;

- Nadir $\mathrm{SaO}_{2}$ mean during sleep;

- Oxygen Desaturation Index, ODI (n desaturation/h sleep);

- TST \% with $\mathrm{SaO}_{2}<90 \%$;

- TST \% with $\mathrm{SaO}_{2}<80 \%$.

\section{ENT evaluation}

Each child underwent ear, nose and throat evaluation characterised by otoscopy, anterior rhinoscopy and oropharyngoscopy with particular attention to febrile episodes per year in the last 3 years. We evaluated haemochrome, antistreptolysin test, erythrocyte sedimentation rate, and creatine phosphokinase for all children. These clinical parameters are important to understand the indication for surgical treatment in children with adeno-tonsillar hypertrophy.

The tonsils were subjectively measured using a grading system. In grade I, the tonsils were hidden in the tonsillar fossa and were barely visible behind the anterior pillars. In grade II, the tonsils were visible behind the anterior pillars and occupied up to $50 \%$ of the pharyngeal space (the distance between the medial borders of the anterior pillars). In grade III, the tonsils occupied between 50 and $75 \%$ of the pharyngeal space. In grade IV, the tonsils occupied more than $75 \%$ of the pharyngeal space.

Adenoids were analysed during rhinofiberoptic evaluation: a grading system for adenoid hypertrophy was created based on the anatomical relationships between the adenoid tissue and the vomer, soft palate, and torus tubaris. The grading is based on the relationship of the adenoids to adjacent structures when the patient is at rest (i.e., when the soft palate is not elevated).

\section{Adenotonsillectomy}

Patients underwent general anaesthesia in oral intubation maintaining supine in the Rose position. Adenoidectomy was performed followed by extracapsular tonsillectomy using cold/hot technique for dissection and cold/hot for homeostasis ${ }^{33,34}$.

This study was conducted according to the World Medical Association Declaration of Helsinki and was retrospectively registered with number 28/2018.

\section{Statistical analysis}

Nonparametric analyses using the Wilcoxon Test were used to evaluate the effects of treatment (pre- and postadenotonsillectomy) in the variables examined: PSQ test scores, VMI test standard scores (VMI, test motor and visual test), PSGs parameters (TIB, SPT, TST, SOL, FRL, SS-h, AWN-h, SE\%, WASO-min, N1-min, N2-min, N3min, REM-min, WASO spt, N1-spt, N2-spt, N3-spt, REM spt，N1-tst，N2-tst，N3-tst，REM-tst，PSQ，AHI，ODI, OD\%, PLMI).

Pearson's correlation was used to assess the mean preoperative and postoperative VMI scores with preoperative and postoperative polysomnographic parameters. The threshold for statistical significance was $\mathrm{p}<0.05$. All statistical analyses were performed with a statistical software package (STATISTICA 8.0, StatSoft Inc.) 


\section{Results}

The data collected before and 6 months after adenotonsillectomy are indicated in Tables III and IV. Table III shows the results of PSQ and VMI tests. There was a significant difference for PSQ parameters, VMI standard and VMI visual test $(\mathrm{p}<0.05)$, but not for the VMI motor test.
In Table IV PSG parameters before and after adenotonsillectomy are shown. There was a significant difference for macrostructural sleep and respiratory parameters TIB, SPT, TST, SOL, SS-h, AWN-h, SE\%, WASO-min, N1-min, N2-min, N3-min, REM-min, WASO spt, N1-spt, N2-spt, N3-spt, N1-tst, N2-tst, N3-tst, PSQ, AHI, ODI, OD\%, PLMI ( $\mathrm{p}<0.05)$.

Table III. Comparison of PSQ and VMI scores before and after adenotonsillectomy.

\begin{tabular}{lccccc} 
& Pre & Post & & \multicolumn{2}{c}{ Wilcoxon Test } \\
& Mean (DS) & Mean (DS) & U & Z & p \\
PSQ & $0.37(0.15)$ & $0.15(0.07)$ & 0.00 & 5.011926 & 0.000001 \\
VMI st & $106.42(17.84)$ & $111.24(13.32)$ & 61.50000 & 4.375023 & 0.000012 \\
VMI motor test st & $103.87(25.39)$ & $114.35(17.67)$ & 60.00000 & 1.679970 & 0.092964 \\
VMI visual test st & $97.78(15.82)$ & $107.43(14.61)$ & 24.00000 & 4.854568 & 0.000001 \\
\hline
\end{tabular}

Table IV. Comparison of PSG parameters before and after adenotonsillectomy.

\begin{tabular}{|c|c|c|c|c|c|c|c|}
\hline & & & & & & Vilcoxon Tes & \\
\hline & Mean & DS & Mean & DS & U & Z & $p$ \\
\hline TIB-min & 386.4057 & 83.37142 & 589.1892 & 86.07090 & 0.00 & 5.302829 & 0.000000 \\
\hline SPT-min & 342.3486 & 64.93097 & 555.4730 & 75.34052 & 0.00 & 5.302829 & 0.000000 \\
\hline TST-min & 269.0622 & 56.76741 & 529.8784 & 70.22715 & 0.00 & 5.302829 & 0.000000 \\
\hline SOL-min & 44.0570 & 43.74958 & 24.6757 & 18.74656 & 211.0000 & 2.119623 & 0.034039 \\
\hline FRL-min & 104.2919 & 31.16452 & 130.0405 & 54.27404 & 220.0000 & 1.983847 & 0.047274 \\
\hline SS-h & 9.7838 & 2.59235 & 7.5243 & 3.41121 & 162.0000 & 2.858851 & 0.004252 \\
\hline AWN-h & 8.7324 & 2.60502 & 1.6486 & 1.86467 & 0.00 & 5.302829 & 0.000000 \\
\hline SE\% & 70.3054 & 9.19560 & 90.2514 & 5.60063 & 3.000000 & 5.257571 & 0.000000 \\
\hline WASO-min & 73.2865 & 23.48791 & 25.5946 & 26.47479 & 23.50000 & 4.862423 & 0.000001 \\
\hline $\mathrm{N} 1-\mathrm{min}$ & 57.1135 & 23.98776 & 17.3514 & 21.95572 & 46.00000 & 4.608860 & 0.000004 \\
\hline N2-min & 88.5378 & 33.91517 & 231.5946 & 46.38110 & 0.00 & 5.302829 & 0.000000 \\
\hline N3-min & 57.7568 & 25.12663 & 162.1622 & 69.83563 & 1.000000 & 5.287743 & 0.000000 \\
\hline REM-min & 65.6541 & 23.08347 & 118.6892 & 32.43141 & 19.00000 & 5.016190 & 0.000001 \\
\hline WAS0-spt & 21.5716 & 6.21865 & 4.4541 & 4.44607 & 2.000000 & 5.272657 & 0.000000 \\
\hline N1-spt & 16.7676 & 6.79174 & 3.1730 & 3.93211 & 9.000000 & 5.167053 & 0.000000 \\
\hline N2-spt & 25.6962 & 8.50912 & 42.1000 & 7.81466 & 12.00000 & 5.121794 & 0.000000 \\
\hline N3-spt & 16.8414 & 6.54840 & 28.6946 & 9.68389 & 31.00000 & 4.835155 & 0.000001 \\
\hline REM-spt & 19.1227 & 6.26117 & 21.5595 & 5.89545 & 245.0000 & 1.606689 & 0.108124 \\
\hline N1-tst & 21.7743 & 9.65647 & 3.4189 & 4.38209 & 8.000000 & 5.182139 & 0.000000 \\
\hline N2-tst & 32.3411 & 9.17601 & 44.0432 & 7.74441 & 63.00000 & 4.352393 & 0.000013 \\
\hline N3-tst & 21.4795 & 8.15687 & 30.0432 & 10.01681 & 106.0000 & 3.703683 & 0.000213 \\
\hline REM-tst & 24.4041 & 7.89300 & 22.5108 & 5.83423 & 284.0000 & 1.018324 & 0.308525 \\
\hline $\mathrm{AHI}$ & 10.3378 & 3.01786 & 5.9216 & 1.30110 & 11.00000 & 5.136880 & 0.000000 \\
\hline ODI & 5.8270 & 1.81974 & 3.5027 & 0.55752 & 12.00000 & 5.043095 & 0.000000 \\
\hline Mean OD\% & 94.4865 & 1.97120 & 96.8000 & 0.73749 & 7.000000 & 5.197225 & 0.000000 \\
\hline Lowest 0D\% & 89.3405 & 2.96287 & 94.3568 & 0.93290 & 0.00 & 5.231621 & 0.000000 \\
\hline Average 0D\% & 5.1459 & 2.34338 & 2.4432 & 0.73543 & 5.000000 & 5.227398 & 0.000000 \\
\hline PLMI & 6.5062 & 2.31283 & 3.1746 & 1.14721 & 29.00000 & 4.865327 & 0.000001 \\
\hline
\end{tabular}


Clinical evaluation at PSQ six months after adenotonsillectomy showed a reduction in values compared with those before surgery (Fig. 2).

VMI standard scores, VMI motor test and VMI visual test scores showed improvements in performance at 6 months after surgery, which was significant for standard and visual scores (Figs. 3, 4) and not significant for motor scores (Fig. 5).

Spearman rank order correlation between preoperative

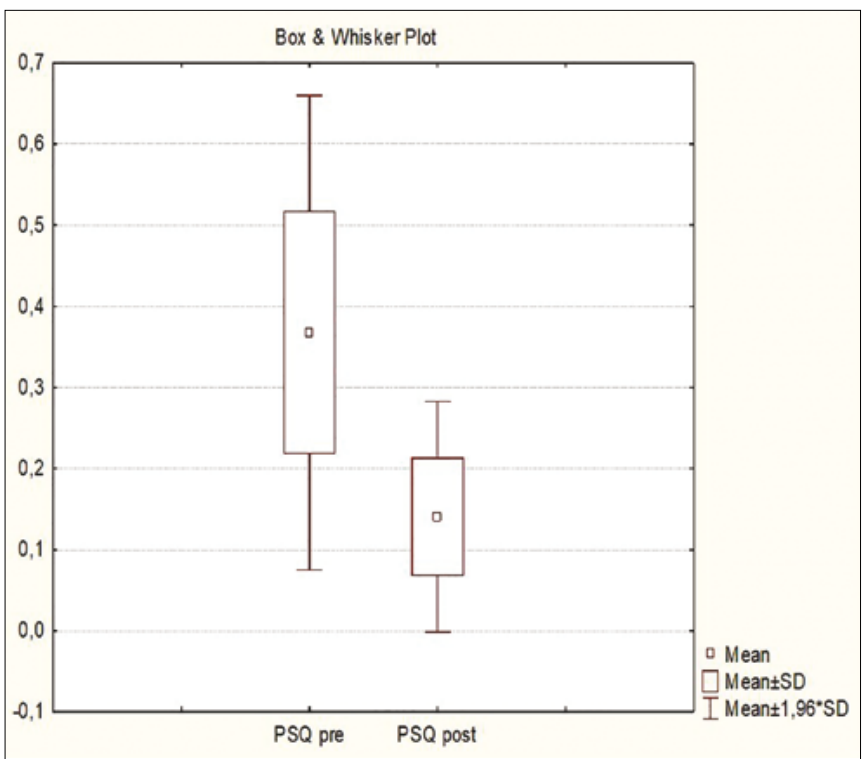

Figure 2. Comparison of PSQ scores before and six months after adenotonsillectomy.

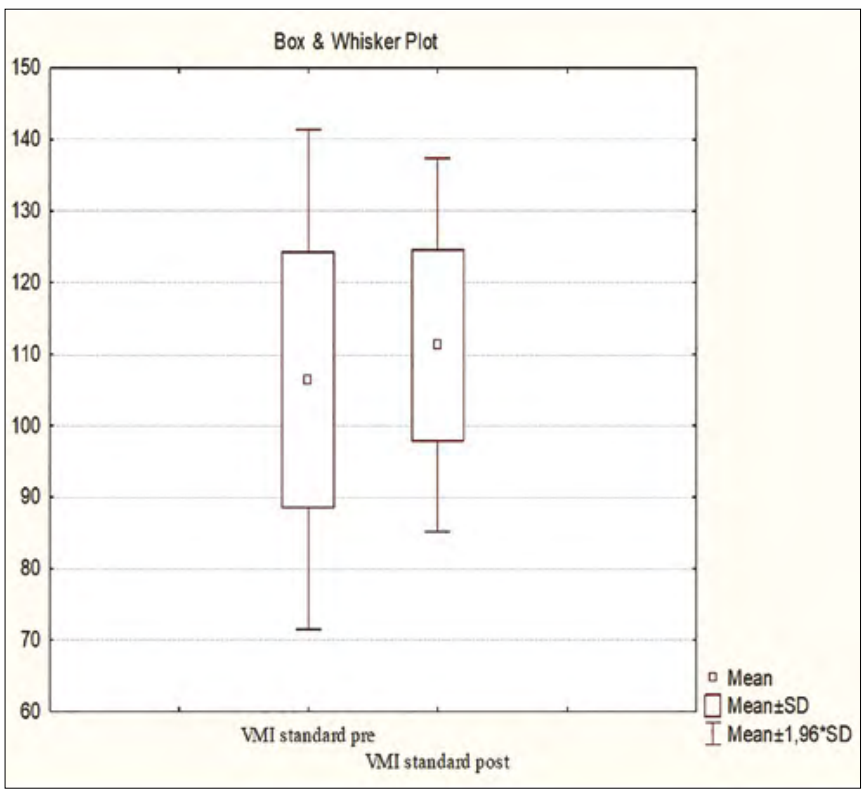

Figure 3. Comparison of VMl standard scores before and six months after adenotonsillectomy (statistically significant). and postoperative VMI scores and preoperative and postoperative PSG parameters was statistically significant $\mathrm{p}<0.05$ (Tab. V): there was no linear correlation. There was a significant difference for PSQ parameters, VMI standard, visual tests scores and PSG parameters, but not for motor test scores before and after adenotonsillectomy in children affected by OSAS. When comparting VMI standard, motor and visual tests score with PSG parameters, the difference before and after surgery was statistically significant.

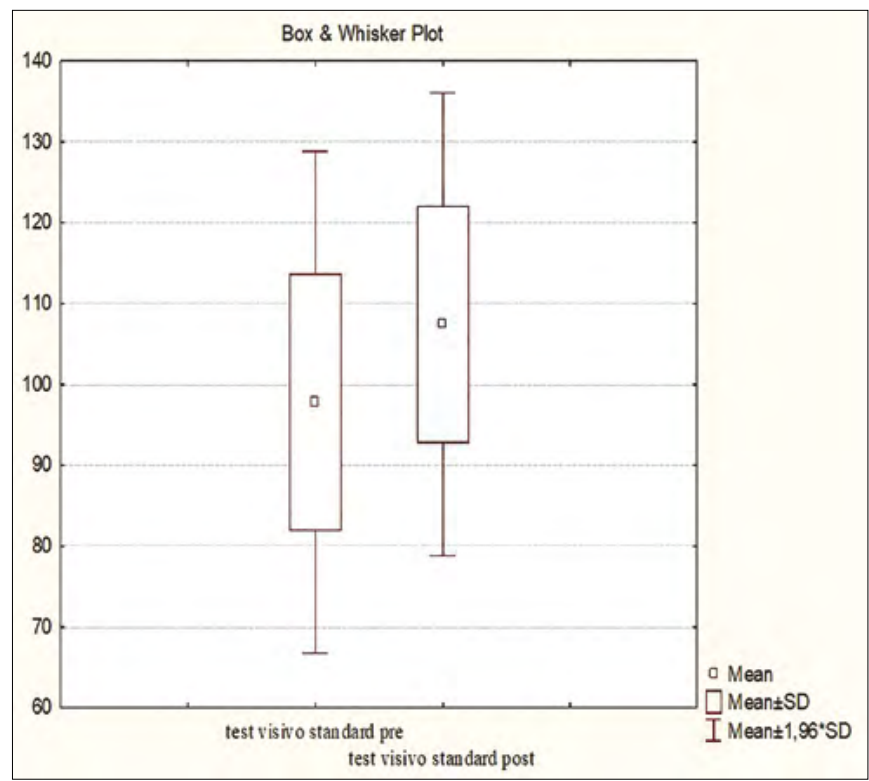

Figure 4. Comparison of VMI visual test scores before and six months after adenotonsillectomy (statistically significant).

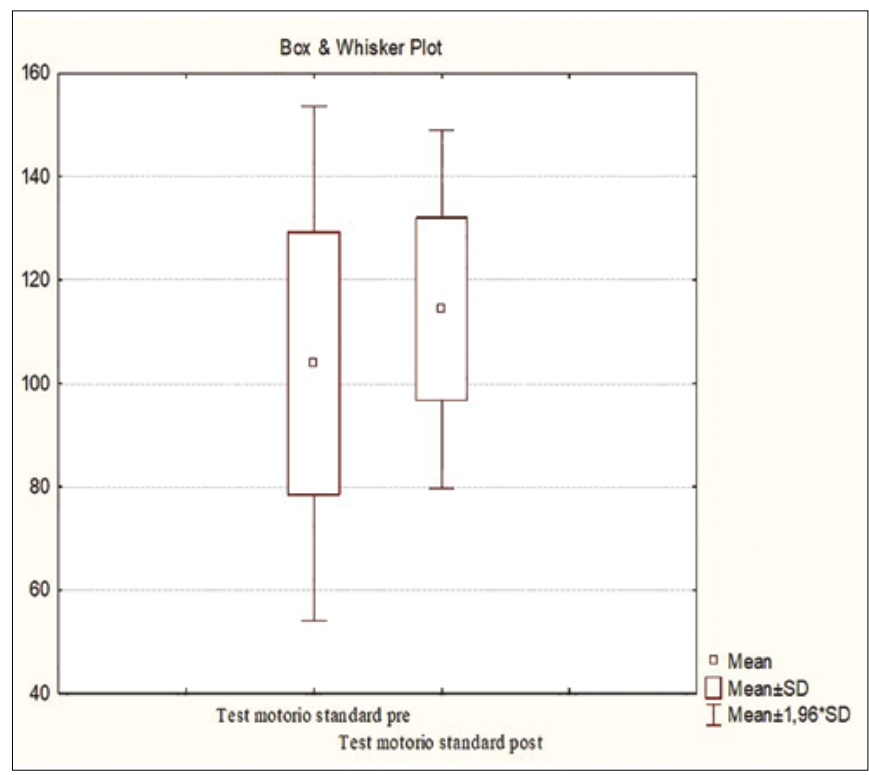

Figure 5. Comparison of VMI motor test scores before and six months after adenotonsillectomy (not statistically significant). 
Table V. Correlation between VMI scores and PSG parameters before and 6 months after adenotonsillectomy.

\begin{tabular}{|c|c|c|c|}
\hline & \multicolumn{3}{|c|}{$\begin{array}{l}\text { Spearman Rank Order Correlations } \\
\text { MD pairwise deleted } \\
\text { Marked correlations are significant at } p<0.05000\end{array}$} \\
\hline & $\begin{array}{l}\text { VMI } \\
\text { st }\end{array}$ & $\begin{array}{l}\text { VMI motor } \\
\text { test st }\end{array}$ & $\begin{array}{l}\text { VMI visual } \\
\text { test st }\end{array}$ \\
\hline TIB-min & 0.144274 & -0.097321 & -0.116585 \\
\hline SPT-min & 0.211697 & -0.059812 & -0.108225 \\
\hline TST-min & 0.167254 & -0.058115 & -0.099394 \\
\hline SOL-min & -0.132756 & -0.016344 & -0.042546 \\
\hline FRL-min & -0.068619 & -0.072612 & 0.044218 \\
\hline SS-h & 0.097221 & -0.171699 & -0.155499 \\
\hline AWN-h & 0.110438 & -0.014303 & -0.214789 \\
\hline SE\% & 0.063701 & 0.049596 & 0.152116 \\
\hline WASO-min & 0.132909 & -0.046434 & -0.173408 \\
\hline N1-min & -0.018648 & 0.093144 & -0.037976 \\
\hline N2-min & 0.099488 & 0.025531 & 0.027575 \\
\hline N3-min & 0.197135 & -0.080507 & -0.121304 \\
\hline REM-min & -0.039059 & -0.070151 & 0.111569 \\
\hline WASO-spt & 0.023792 & -0.038759 & -0.101054 \\
\hline N1-spt & -0.053335 & 0.119138 & 0.011890 \\
\hline N2-spt & -0.035937 & 0.064147 & 0.116147 \\
\hline N3-spt & 0.170595 & -0.085442 & -0.132817 \\
\hline REM-spt & -0.203929 & -0.072725 & 0.220432 \\
\hline N1-tst & -0.055982 & 0.096213 & 0.001882 \\
\hline N2-tst & -0.040447 & 0.066204 & 0.088461 \\
\hline N3-tst & 0.194365 & -0.082518 & -0.158518 \\
\hline REM-tst & -0.173607 & -0.081733 & 0.147272 \\
\hline PSQ & -0.121546 & 0.035007 & -0.021625 \\
\hline AHI & -0.084110 & 0.144959 & 0.085684 \\
\hline ODI & -0.011723 & 0.134956 & -0.076610 \\
\hline Mean 0D\% & -0.006353 & 0.062628 & -0.027591 \\
\hline Lowest 0D\% & 0.012973 & -0.005733 & 0.034711 \\
\hline Average 0D\% & -0.019340 & 0.040488 & -0.044393 \\
\hline PLMI & 0.026528 & -0.054086 & -0.021249 \\
\hline
\end{tabular}

\section{Discussion}

Diagnosis and treatment of suspected mechanical OSAS in children represent a highly controversial issue for the differences in terms of diagnostic resources and therapeutic approach. Only careful evaluation of medical history and clinical presentation together with polysomnography, can provide the right diagnostic classification and, consequently, the correct choice of treatment ${ }^{35-38}$. The resolution of mechanical obstruction must be as fast as possible in order to reduce the risk of cardiac, metabolic and neurological complications ${ }^{7,8}$. Our attention is focused on the close relationship between OSAS and neurocognitive and neurobehavioral disorders in very young children with a mean age of $8.44 \pm 2.26$. The set of symptoms referred by patients or by children's parents together with respiratory distress is often characterised by attention deficit and hyperactivity, irritability and learning disorders ${ }^{9-12}$. Recent studies have shown a clear relationship of cause and effect between nocturnal hypoxia leading to respiratory events (obstructive apnoeas and hypopnoeas), alteration of the structure of sleep with fragmentation and arousals and neurocognitive deficit ${ }^{9-12}$. In particular, it has been established that in OSAS patients the brain area mostly involved in the hypo-anoxic stress is the prefrontal cortex ${ }^{9,38}$. It presents a considerable reduction in activity in all stages of sleep and seems to be disconnected from other cortex areas ${ }^{9,37}$. Many authors have shown considerable improvement after adenotonsillectomy in children's cognitive performance (memory, learning, IQ) and in quality of life (reduced daytime sleepiness, irritability and mood alteration) ${ }^{15,38}$. These authors have used: Differential Ability Scales (similar to I.Q.), Non Verbal Cluster ${ }^{36}$; Behavioral Assessment System for Children ${ }^{39}$ (BASC), to evaluate the behaviour (mood, hyperactivity and somatisation); Epworth sleepiness Stairs and Osler Test (to evaluate semantic, episodic and work memory) ${ }^{15}$; the Standfort Binet Intelligence Scale 5th edition and the Developmental Neuropsychological Assessment (NEPSY) for neuropsychological skills ${ }^{40}$.

VMI test has never been performed to evaluate visualmotor performances in children with OSAS.

The Beery-Buktenuica Developmental Test of Visual-Motor Integration (VMI; Beery \& Beery, 2004) was developed to assess visuoperceptual and constructional abilities in children and adolescents and is among the most widely administered neuropsychological tests ${ }^{32,41,42}$. This test has been used to evaluate children with traumatic brain injury and attention-deficit/hyperactivity disorder (ADHD) ${ }^{42}$. We evaluated children with OSAS using VMI standard scores, motor and visual test scores, PSQ and PSG parameters before and at 6 months after adenotonsillectomy. Visuoperceptual and constructional performances were improved in all children after surgical treatment; analysis of sleep quality (respiratory parameters and neurological ones) showed better sleep macrostructural architecture and better respiratory scores after adenotonsillectomy than before.

The results showed the achievement of therapeutic benefits. Six months after the mechanical removal of the obstruction there was an improvement of cognitive performance and quality of life.

\section{Conclusions}

Dealing with a subject so controversial for the lack of homogeneity in terms of diagnostic and therapeutic 
resources, such as OSAS in children, we chose a multidisciplinary approach. Nowadays there are no studies in the literature specifically correlating mechanical OSAS and visual-spatial skills. We first assessed the deficiencies and then the improvements in visual-spatial skills in children affected by OSAS before and after adenotonsillectomy. Our idea has been confirmed by the results obtained from the 6 month postoperative VMI tests. At any starting level, all children showed improvement in performance. Thus, at 6 months after adenotonsillectomy important therapeutic benefits have been demonstrated both in visual-motor performance and in the child's quality of life according to the objective data of PSG.

\section{References}

1 Lumeng JC, Chervin RD. Epidemiology of pediatric obstructive sleep apnea. Proc Am Thorac Soc 2008;5:242-52. https://doi.org/10.1513/ pats.200708-135MG

2 Castronovo V, Zucconi M, Nosetti L, et al. Prevalence of habitual snoring and sleep-disordered breathing in preschool-aged children in an Italian community. J Pediatr 2003;142:377-82. https://doi. org/10.1067/mpd.2003.118

3 American Academy of Pediatrics Section on Pediatric Pulmonology. Clinical Practice guideline: diagnosis and management of childhood obstructive sleep apnea syndrome. Pediatrics 2002;109:704-12. https://doi.org/10.1542/peds.109.4.704

4 Ali NJ, Stradling JR. Epidemiology and natural history of snoring and sleep-disordered creathing in children. In: Loughlin GM, Carrol JL, Marcus CL (eds.). Sleep and breathing in children: a developmental approach. New York: Marcel Dekker; 2000. pp. 555-74.

5 Rosen CL. Obstructive sleep apnea syndrome in children: controversies in diagnosis and treatment. Pediatr Clin North Am 2004;51:15367. https://doi.org/10.1016/s0031-3955(03)00183-4

6 Diagnostic Classification Steering Committee. International classification of sleep disorders: diagnostic and coding manual. Rochester, MN: American Sleep Disorders Association; 1990.

7 O'Brein LM, Gozal D. Neurocognitive dysfunction and sleep in children: from human to rodent. Pediatr Clin N Am 2004;51:187-202. https://doi.org/10.1016/s0031-3955(03)00184-6

8 Lipton JL, Gozal D. Treatment of obstructive sleep apnea in children: do you really know how? Sleep Med Rev 2003;7:61-80. https://doi. org/10.1053/smrv.2001.0256

9 Goldstein NA, Fatima M, Campbell TF, et al. Child behavior and quality of life before and after tonsillectomy and adenoidectomy. Arch Otolaryngol Head Neck Surg 2002;128:770-5. https://doi. org/10.1001/archotol.128.7.770

$10 \mathrm{Li}$ AM, Au CT, So HK, et al. Prevalence and risk factors of habitual snoring in primary school children. Chest 2010;138:519-27. https:// doi.org/10.1378/chest.09-1926

11 Wise MS, Nichols CD, Grigg-Damberger MM, et al. Executive summary of respiratory indications for polysomnography in children: an evidence-based review. Sleep 2011;34:389-98. https://doi. org/10.1093/sleep/34.3.389

12 Spruyt K, O'Brein LM, Coxon APM, et al. Multidimentional scaling of pediatric sleep breathing problems and bio-behavioral correlates. Sleep Med 2006;7:269-80. https://doi.org/10.1016/j. sleep.2005.08.013
13 Amin RS, Kimball TR, Bean JA, et al. Left ventricular hypertrophy and abnormal ventricular geometry in children and adolescents with obstructive sleep apnea. Am J Respir Crit Care Med 2002;165:13959. https://doi.org/10.1164/rccm.2105118

14 Sofer S, Weinhouse E, Tal A, et al. Cor pulmonale due to adenoidal or tonsillar hypertrophy oe both in children. Non invasive diagnosis and follow-up. Chest 1988;93:119-22. https://doi.org/10.1378/ chest.93.1.119

15 Waters KA, Cheng ATL. Adenotonsillectomy in the context of sleep apnoea. Paediatr Respir Rev 2009;10:25-31. https://doi.org/10.1016/j. prrv.2008.10.002

16 Kaditis AG, Alonso Alvarez ML, Boudewyns A, et al. Obstructive sleep disordered sleeping in 2- to 18-year-old children: diagnosis and management. Eur Respir J 2016;47:69-94. https://doi. org/10.1183/13993003.00385-2015

17 Kaditis A, Kheirandish-Gozal L, Gozal D. Algorithm for the diagnosis and treatment of pediatric OSA: a proposal of two pediatric sleep centers. Sleep Med 2012;13:217-27. https://doi.org/10.1016/j. sleep.2011.09.009

18 Marcus CL, Moore RH, Rosen CL, et al. A randomized trial of adenotonsillectomy for childhood sleep apnea. $\mathrm{N}$ Engl J Med 2013;368:2366-76. https://doi.org/10.1056/NEJMoa1215881

19 Goodwin JL, Vasquez MM, Silva GE, et al. Incidence and remission of sleep disordered breathing and related symptoms in 7- to 17- years old children - the Tucson children assessment of sleep apnea study. J Pediatr 2010;157:57-61. https://doi.org/10.1016/j.jpeds.2010.01.033

20 Marcus CL, Brooks LJ, Draper KA, et al. Diagnosis and management of childhood obstructive sleep apnea syndrome. Pediatrics 2012;130:e714-55. https://doi.org/10.1542/peds.2012-1672

21 Motta G, Motta S, Cassano P, et al. Effects of guidelines on adenotonsillar surgery on the clinical behavior of otorhinolaryngologists in Italy. BMC Ear Nose Throat Disord 2013;13:1. https://doi. org/10.1186/1472-6815-13-1

22 Accardo JA, Shults J, Leonard MB, et al. Differences in overnight polysomnography scores using the adult and pediatric criteria for respiratory events in adolescents. Sleep 2010;33:1333-9. https://doi. org/10.1093/sleep/33.10.1333

23 Iber C, Ancoli-Israel S, Chesson A, et al. AASM Manual for the scoring of sleep associated events: rules, terminology and technical specifications. First Edition. Westchester, Illinois: American Academy of Sleep Medicine; 2007.

24 Cohen SR, Lefaivre JF, Burstein FD, et al. Surgical treatment of obstructive sleep apnea in neurologically compromised patients. Plast Reconstr Surg 1997;99:638-46. https://doi.org/10.1097/00006534199703000-00005

25 Julliand S, Boule M, Baujat G, et al. Lung function, diagnosis, and treatment of sleep-disordered breathing in children with achondroplasia. Am J Med Genet A 2012;158A:1987-93. https://doi.org/10.1002/ ajmg.a.35441

26 Addo NK, Javadpour S, Kandasamy J, et al. Central sleep apnea and associated Chiari malformation in children with syndromic craniosynostosis: treatment and outcome data from a supraregional national craniofacial center. J Neurosurg Pediatr 2013;11:296-301. https://doi. org/10.3171/2012.11.PEDS12297

27 Bourke RS, Anderson V, Yang JSC, et al. Neurobehavioral function is impaired in children with all severities of sleep disordered breathing. Sleep Med 2011;12:222-9. https://doi.org/10.1016/j. sleep.2010.08.011

28 Jackman AR, Biggs SN, Walter LM, et al. Sleep-disordered breathing in preschool children is associated with behavioral, but not cognitive, impairments. Sleep Med 2012;13:621-31. https://doi.org/10.1016/j. sleep.2012.01.013 
29 Yuan HC, Sohn EY, Abouezzeddine T, et al. Neurocognitive functioning in children with obstructive sleep apnea syndrome: a pilot study of positive airway pressure therapy. J Pediatr Nursing 2012;27:607-13. https://doi.org/10.1016/j.pedn.2011.07.007

30 Gozal D. Obstructive sleep apnea in children: implications for the developing central nervous system. Semin Pediatr Neurol 2008;15:100-6. https://doi.org/10.1016/j.spen.2008.03.006

31 Miano S, Paolino MC, Urbano A, et al. Neurocognitive assessment and sleep analysis in children with sleep-disordered breathing. Clin Neurophysiol 2011;122:311-9. https://doi.org/10.1016/j. clinph.2010.06.019

32 Beery KE, Beery NA. The beery-buktenica developmental test of visual-motor integration: administration, scoring, and teaching manual. Fifth Edition. Minneapolis, MN: NCS Pearson; 2006.

33 Motta S, Testa D, Ferrillo B, et al. Can a surgical technique be a risk for post-tonsillectomy haemorrhage? Our point of view. Arch Otolaryngol Head Neck Surg 2018;1:4. https://doi.org/10.24983/ scitemed.aohns.2018.00057

34 Motta S, Testa D, Ferrillo B, et al. Surgical techniques and posttonsillectomy haemorrhage. Curr Pediatr Res 2017;21:559-66.

35 Rosen CI. Obstructive apnea syndrome (OSAS) in children: diagnostic challenges. Sleep 1996;19:274-7.

36 Marcus CL. Sleep-disordered breathing in children. Curr Opin Pediatr 2000;12:208-12. https://doi.org/10.1097/00008480-200006000-00005
37 Guillelminault C, Akhtar F. Pediatric sleep-disordered breathing: new evidence on its development. Sleep Med Rev 2015;24:46-56. https:// doi.org/10.1016/j.smrv.2014.11.008

38 Rosen CL, Morton S, Larkin E, et al. Persistence of sleep disordered breathing in children post-tonsillectomy. Am J Respir Crit Care Med 2001;163:A184.

39 Mitchell B, Kelly J. Behavioral changes in children with mild sleep-disordere breathing or obstructive sleep apnea after adenotonsillectomy. Laryngoscope 2007;117:1685-8. https://doi. org/10.1097/MLG.0b013e318093edd7

40 Kholer MJ,Lushington K, van den HeuvelCJ, et al. Adenotonsillectomy and neurocognitive deficits in children with sleep disordered breathing. PLoS One 2009;4:e7343. https://doi.org/10.1371/journal. pone. 0007343

41 Rabin LA, Barr WB, Burton LA. Assessment practicies of clinical neuropsychologists in the United States and Canada: a survey of INS, NAN, and APA Division 40 members. Arch Clin Neuropsychol 2005;20:33-65. https://doi.org/10.1016/j.acn.2004.02.005

42 Sutton GP, Bachard KA, Bello DT, et al. Beery-buktenica developmental test of visual-motor integration performance in children with traumatic brain injury and attention-deficit/hyperactivity disorder. Psycolog Assess 2011;23:805-9. https://doi.org/10.1037/ a0023370 